\title{
EchoGéo
}

54 | 2020

Varia

\section{Shanzhai et culture du faire en Chine}

Vers un nouveau modèle d'innovation numérique autour de Shenzhen?

Shanzhai and maker culture in China: Towards a new digital innovation model in Shenzhen's area?

\section{Julia Nawrocki}

\section{OpenEdition}

\section{Journals}

\section{Édition électronique}

URL : https://journals.openedition.org/echogeo/20383

DOI : 10.4000/echogeo.20383

ISSN : 1963-1197

\section{Éditeur}

Pôle de recherche pour l'organisation et la diffusion de l'information géographique (CNRS UMR 8586)

\section{Référence électronique}

Julia Nawrocki, « Shanzhai et culture du faire en Chine », EchoGéo [En ligne], 54 | 2020, mis en ligne le 31 décembre 2020, consulté le 26 août 2021. URL : http://journals.openedition.org/echogeo/20383 ; DOI : https://doi.org/10.4000/echogeo.20383

Ce document a été généré automatiquement le 26 août 2021.

EchoGéo est mis à disposition selon les termes de la licence Creative Commons Attribution - Pas d'Utilisation Commerciale - Pas de Modification 4.0 International (CC BY-NC-ND) 


\section{Shanzhai et culture du faire en Chine}

Vers un nouveau modèle d'innovation numérique autour de Shenzhen?

Shanzhai and maker culture in China: Towards a new digital innovation model

in Shenzhen's area?

Julia Nawrocki

\section{Introduction}

1 Dans les années 1960, l'économiste Fritz Machlup établissait le concept de l'économie de la connaissance, en constatant qu'aux Etats-Unis, la dimension immatérielle du travail devenait centrale dans la création de valeur. Aujourd'hui, « les frontières entre économie de la connaissance, économie culturelle, ou économie créative se chevauchent » (Liefooghe, 2010), ces termes étant repris dans tous les discours sur la modernisation économique, et certains auteurs suggèrent l'avènement d'une nouvelle phase du capitalisme. Elle succèderait à celles des capitalismes mercantiliste puis industriel, et aurait été permise par la mobilisation des nouvelles technologies de l'information et des communications. Ce capitalisme cognitif aurait la particularité d'être basé sur une « force de travail capable de maximiser la capacité d'apprentissage, d'innovation et d'adaptation à une dynamique de changement continu " (Monnier et Varcellone, 2014), où le temps de travail s'étendrait à l'ensemble du temps de vie (Lazzarato et Negri, 1991). Puisque l'innovation fonde la compétitivité des entreprises, celles-ci privilégient la créativité et la polyvalence de leurs employés, et tentent de mobiliser des compétences acquises hors du temps de travail salarié. Elles intègrent alors des pratiques telles que le travail en code source ouvert, issu de la contreculture hackeur qui défendait pourtant un mode de production libre de droits, alternatif au capitalisme dominant. Pour les territoires, l'un des enjeux est donc d'attirer les talents, de leur offrir un cadre favorable à l'épanouissement de leur créativité, entrainant l'élaboration de divers modèles de villes créatives ou de villes innovantes qui concentrent la création de valeur et les entreprises. 
2 Cette transition vers une économie fondée sur l'innovation, qui serait la clé du développement, s'inscrit dans un contexte où les technologies numériques tiennent une place croissante dans nos sociétés. En 1965 puis en 1975, Gordon E. Moore, l'un des fondateurs d'Intel, établit deux conjectures sur l'évolution de la puissance de calcul des ordinateurs et de la complexité du matériel informatique, d'après lesquelles le nombre de transistors des microprocesseurs sera amené à doubler tous les deux ans. Ces observations, que l'on a ensuite appelées les «lois de Moore ", ont ancré l'idée d'une augmentation exponentielle de la vitesse des ordinateurs, et par extension, d'une accélération tout aussi rapide du progrès technique. Pour ce faire, les idées devront être concrétisées le plus vite possible, grâce à un écosystème productif efficace qui sélectionnera ensuite les idées intéressantes.

En Chine, c'est la ville de Shenzhen qui a été choisie pour représenter ce nouveau modèle industriel. Le premier ministre chinois Li Keqiang, s'y est rendu en 2015 pour promouvoir la politique «internet + » du $13^{\text {ème }}$ plan quinquennal. Il parle alors d'un modèle du Created in China qui remplacerait celui du Made in China, dont l'essor avait largement été permis par la mise à profit d'une main-d'œuvre abondante et bon marché, une politique active d'aménagement du territoire et l'ouverture de zones économiques spéciales.

Illustration - 1- La zone économique spéciale de Shenzhen dans le delta de la Rivière des Perles

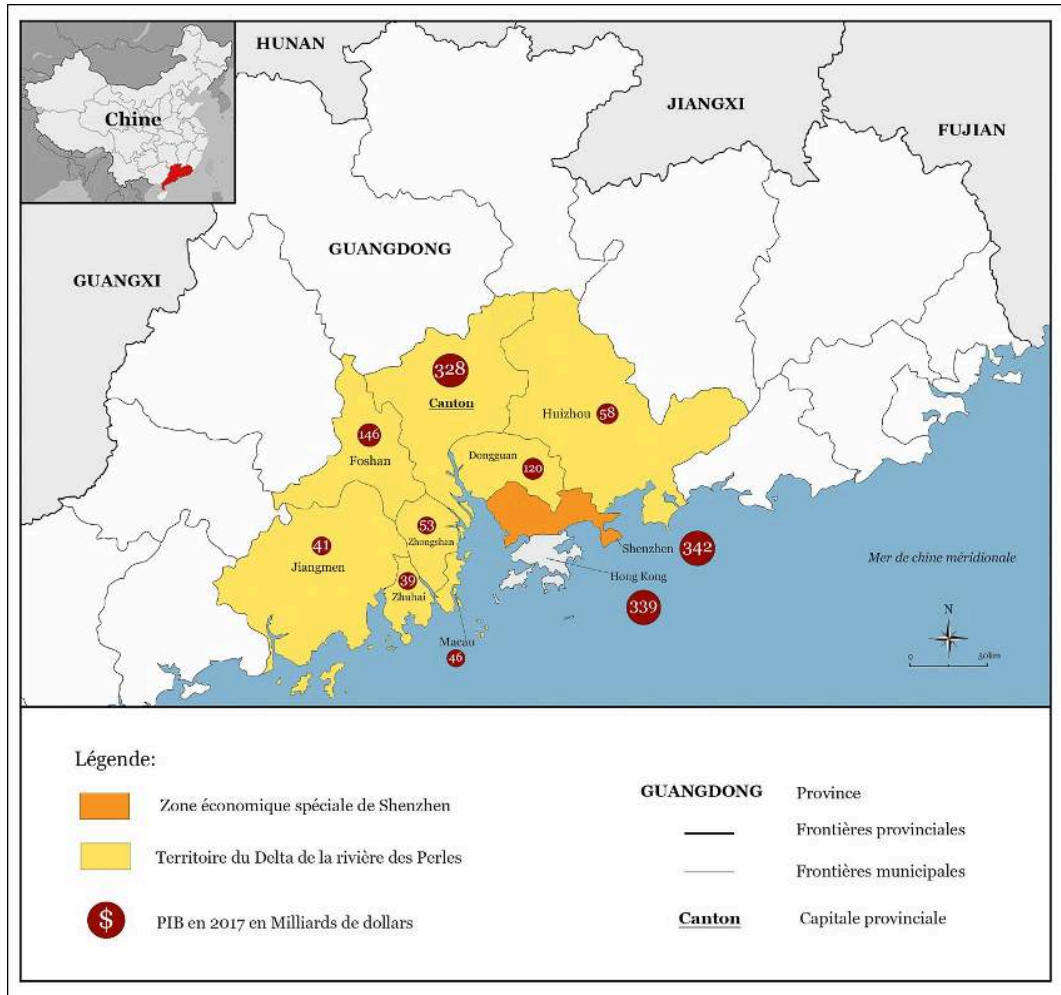

Source : Municipal Bureau of Statistics, IMF 2017. Auteur : J. Nawrocki, 2020.

4 Au cours des dernières décennies, de nombreuses entreprises mondiales ont délocalisé leur production vers ces zones qui sont devenues de grands bassins manufacturiers. Parmi elles, Shenzhen est une de ces métropoles chinoises qui revendiquent actuellement le statut de ville mondiale de l'innovation. Connue comme le «Petit village de pêcheurs » au début des années 1980, son PIB dépasse désormais celui de 
Hong Kong, atteignant 342 milliards de dollars en 2017, avec un taux de croissance de $7,5 \%$ du PIB contre $3 \%$ à Hong Kong. Shenzhen a connu depuis les années 2010 une popularisation rapide et massive de la culture occidentale hackeur, l'ouverture et la promotion de nombreux makerspaces, des espaces de fabrication numérique communautaires, tout en disposant de cette organisation industrielle régionale héritée de la mondialisation et des investissements étrangers de la fin du XX ${ }^{\text {ème }}$ siècle. S'y est construit un discours original inspiré des pratiques en code source ouvert (open-source) et influencé par le contexte local, dont l'étude pourrait permettre de mieux comprendre les liens entre territoire et innovation numérique.

5 L'innovation est souvent étudiée en rapport avec un cadre libéral, non seulement économique mais aussi politique (Keane, 2004, 2006; O'Connor et Xin, 2006; Florida, 2002). À travers la figure de l'artiste subversif ou de l'entrepreneur créatif, elle est associée à des valeurs telles que la liberté de pensée ou la liberté d'expression, ainsi qu'à une certaine forme d'individualisme aux racines des démocraties occidentales. Les technologies numériques sont quant à elles trop souvent perçues comme une suite d'avancées techniques naturelles, inévitables, analysées dans leur environnement scientifique mais détachées de tout contexte culturel et spatial. En plus d'être supposément neutres, elles seraient le support de ce que l'on a appelé le "Cyberespace ", d'après le roman de science-fiction Neuromancien de William Gibson, monde informationnel dont on oublie souvent qu'il repose sur une infrastructure matérielle, et qu'on appréhende comme s'il était «doté de propriétés distinctes de celles du monde physique ». Pour Sébastien Broca, ce lieu commun, ainsi que celui de la "déterritorialisation, qui présente les collectifs en ligne comme détachés de tout ancrage territorial [...] contribuent à occulter la continuité entre les transformations du monde numérique et celles du reste de la société » (Broca, 2018).

C'est donc cette continuité que cet article propose de questionner, à travers l'analyse de la culture du faire de Shenzhen et de son organisation au sein d'un écosystème innovant qui se présente désormais comme un modèle alternatif à celui de l'Occident. Il est le fruit d'un travail de terrain effectué en 2019 dans le cadre d'un master de géographie, durant lequel ont été menés des entretiens semi-directifs avec les principaux acteurs $\mathrm{du}$ mouvement du matériel libre (open-hardware), tels que les fondateurs de onze différents espaces de fabrication numérique, leurs membres, ainsi que des entrepreneurs et des ingénieurs gravitant autour de la scène du faire de Shenzhen. Cette recherche s'appuie également sur trois mois d'observation participante dans les espaces Troublemaker et Steamhead, autour de projets centrés sur l'apprentissage de la fabrication et de la programmation informatique. Surtout, elle poursuit les travaux commencés par Silvia Lindtner, David Li, Clément Renaud, Florence Graezer Bideau et Marc Laperrouzza sur ces nouvelles pratiques numériques à Shenzhen (Lindtner et al., 2015 ; Renaud, 2017 ; Fernandez et al., 2016 ; Graezer Bideau et Renaud, 2020), tout en se fondant sur des recherches en géographie (Sanjuan, 1997) et en économie (Arvanitis et al., 2006, 2005; Wu, 2016) menées dans la région, ainsi que sur les apports des sciences de l'information et de la communication (Broca, 2013 et 2018; Arrivé, 2019). Avec un positionnement pluridisciplinaire, ce cas d'étude entend donc illustrer les liens réciproques entre la production de l'espace géographique et celle des nouvelles technologies. Il interrogera également la notion d'innovation au sein d'un État autoritaire, dans un contexte mondial de séparation des phases de conception et d'assemblage où la connaissance, les données et la créativité sont au cœur de la 
production de valeur. Cet article sera donc aussi une contribution à la littérature managériale concernant les marchés émergents, l'innovation dite inversée (Radjou et al., 2012 ; Hussler, 2015 ; Haudeville et Le bas, 2016) et les « nouvelles logiques spatiales de diffusion des idées » (Hussler, 2015), en montrant comment le pouvoir chinois a su tirer parti de pratiques initialement subversives pour les intégrer au sein d'un même territoire productif.

\section{Constitution d'un territoire manufacturier unique}

\section{Des origines du mouvement hackeur à l'essor des espaces de fabrication numériques}

7 Le terme hackeur, fréquemment utilisé dans le domaine de la cyber-sécurité, est en réalité issu d'un mouvement créé par les informaticiens du laboratoire d'intelligence artificiel du MIT aux États-Unis (AI Lab), dans les années 1970. La diffusion des logiciels n'était alors qu'un moyen de faciliter la vente du matériel informatique qui constituait la principale source de revenus, avant que les éditeurs ne se mettent à vendre des licences d'exploitation. En réaction à ce changement, les membres du AI Lab se sont regroupés autour de Richard Stallman et de son projet de système d'exploitation libre GNU pour défendre l'ouverture des logiciels, et plus précisément l'accès au code source, afin de permettre une libre circulation des connaissances et des innovations informatiques. Stallman fonde alors la Free Software Fondation, définit la notion de « logiciel libre » et crée avec son ami et juriste Eben Moglen la Licence Publique Générale GNU. Celle-ci fixe les conditions de distribution des logiciels du projet GNU, et plus largement, la notion subversive de gauche d'auteur (copyleft), par opposition au droit d'auteur (copyright), pour garantir juridiquement la liberté d'utiliser, de modifier et de diffuser le logiciel ou ses versions dérivées, au cœur de la philosophie du logiciel libre.

8 L'histoire du développement de ce système d'exploitation entraina toutefois l'appropriation de ces pratiques collaboratives par les entreprises ainsi qu'une rupture entre la philosophie du logiciel libre où les performances technologiques restaient secondaires par rapport aux enjeux sociaux du libre et les partisans d'une ouverture du code moins contraignante que la licence GNU (Broca, 2013). Sans adhérer aux idées de Richard Stallman et à son opposition aux logiciels propriétaires, c'est un informaticien du nom de Linus Torvalds qui réussit à résoudre le problème de programmation du noyau manquant au système, qui deviendra GNU/Linux, après l'avoir soumis en ligne à l'ensemble de la communauté des programmeurs (Broca, 2018). Progressivement, les entreprises ont réalisé les avantages commerciaux que pouvait représenter cette innovation collective, décentralisée et gratuite. Aujourd'hui, de nombreuses entreprises comme Google ou IBM développent leurs produits à partir de logiciels libres tel que GNU/Linux avant de les commercialiser sous la protection de droits de propriété intellectuelle, faisant ainsi de l'ouverture du code source un nouveau moyen d'innovation de masse.

9 Parallèlement à la diffusion des pratiques hackeurs dans le monde industriel, le mouvement s'est incarné dans des lieux spécifiques, en anglais les hackerspaces, permettant à la communauté de se rencontrer physiquement pour collaborer, tout en influençant le domaine de la production matérielle à travers le mouvement du faire et ses espaces de fabrication numérique. Proches de la philosophie du logiciel libre, ceux 
qui s'appellent les makers, défendent l'ouverture du code et des procédés de fabrication des machines pour permettre aux usagers de voir, de comprendre la technique et donc d'en reprendre le contrôle. C'est une vision de la société dans laquelle la production est basée sur l'entraide, le partage et où le travail d'innovation reste un loisir. Mettant à disposition les outils nécessaires à la fabrication, l'espace physique prend ici tout son sens et, comme dans le domaine du logiciel, les idées du matériel libre ont vite essaimé dans les milieux universitaires puis industriels. À côté des espaces de fabrication autogérés existent donc les laboratoires du faire (fablabs), financés par des universités ou des entreprises et qui adhèrent à la charte officielle du MIT rédigée dans le cadre d'un cours de Neil Gershenfeld dans les années 2000, ainsi que différents espaces de fabrication rattachés à des entreprises d'accélération ou d'incubation de «jeunes pousses ».

À l'instar du logiciel libre, qui tout en proposant une alternative à l'économie capitaliste actuelle a fini par en inspirer les nouveaux modèles managériaux, le mouvement du matériel libre a été intégré au système productif dominant. À la différence toutefois de la conception logicielle, où le code peut être facilement partagé puis modifié par des millions d'utilisateurs par une simple commande de copier/coller, le matériel électronique ne peut être diffusé et répliqué aussi facilement. Ce contraste explique que le mouvement du faire intéresse encore surtout des passionnés d'informatique et de bricolage technologique et qu'il n'ait pas été absorbé par la production industrielle de masse. En Chine, et principalement à Shenzhen, ce mouvement a cependant pris une ampleur inégalée, du fait de la rencontre des pratiques en code source ouvert avec une culture de la contrefaçon locale, elle-même appuyée sur un bassin manufacturier régional et grâce à un soutien actif du gouvernement.

\section{La culture du libre et la promotion des makerspaces dans un État d'économie planifiée}

11 Le premier espace de fabrication chinois, Xinchejian (新车间), n'ouvre qu'en 2010 à Shanghai (Lindtner et Li, 2012; Saunders et Kingsley, 2016), alors que plus de deux cents autres lieux du même type sont déjà en activité dans le monde (Moilanen, 2012). Très vite, un mouvement du faire se forme autour de six lieux dans les villes de Nanjing, Shanghai, Beijing, Hangzhou, Shenzhen, et Harbin. En 2012 les membres qui ont vécu à l'étranger organisent le premier évènement public destiné à promouvoir la culture hackeur, le Beijing Maker Carnival. La même année, Eric Pan, le fondateur de l'espace de fabrication de Shenzhen Chaihuo, décide d'héberger la première édition du Shenzhen Maker Fair après avoir assisté à trois événements similaires aux États-Unis. Ces deux festivals, qui se tiennent désormais régulièrement et attirent des visiteurs du monde entier, ont contribué à populariser le mouvement du matériel libre en Chine ainsi qu'à attirer l'attention du gouvernement central sur ce mouvement initialement marginal.

Suite à la crise financière de 2008 et face au constat des limites d'un modèle basé sur l'exportation de produits à forte densité de main-d'œuvre peu qualifiée, les autorités chinoises ont décidé de faire du pays le leader mondial en matière d'innovation technologique. Le $13^{\text {ème }}$ plan quinquennal, prévu pour 2016-2020, a donc fixé de nouveaux objectifs, dont «le développement de l'économie créative chinoise et de sa 
capacité innovatrice à partir de la base " (Yu, 2017). C'est dans le cadre de cette politique que s'inscrivait en janvier 2015 la visite du premier ministre chinois Li Keqiang à l'espace de fabrication Chaihuo de Shenzhen, alors que le gouvernement voyait dans le succès du mouvement du faire une opportunité pour démocratiser l'innovation et la production de nouvelles technologies, ainsi qu'un moyen de soutenir les «jeunes pousses » et l'entrepreneuriat. En détournant l'idéologie du faire visant à rendre la technologie et la fabrication accessible à tous, les autorités ont décidé de se servir de ces espaces pour créer un système d'innovation de masse capable de porter un modèle de développement proprement chinois, sous le label Created in China. Alors que le mouvement du faire était né en Occident en tant que contre-culture dans une économie de service, en Chine, le gouvernement y voyait le prolongement d'une industrie d'assemblage à faible valeur ajoutée destinée à l'exportation.

Dans le cadre de cette nouvelle politique nationale, baptisée 众创空间zhongchuang kongjian (littéralement «nombreux espaces de fabrication » ou « espaces de fabrication pour les masses»), des fonds ont été mis à disposition des autorités locales pour subventionner la création d'espaces de fabrication, de laboratoires du faire et d'incubateurs de «jeunes pousses" d'entreprises. D'après les données officielles du ministère des Sciences et des technologies telles que relayées par des médias chinois comme Xinhuanet ou Chinadaily, le nombre de ces espaces de fabrication numérique en Chine serait ainsi passé d'une centaine en 2015 à 4298 à la fin de l'année 2016.

Selon une étude du British Council intitulée Makerspaces and the search for mass innovation (Saunders et Kingsley, 2016), le nombre d'espaces pouvant réellement être labélisés comme espaces de fabrication numérique n'approcherait finalement que les 200 en 2016. Bien que ce nombre ait doublé entre 2015 et 2016, alors qu'il n'en existait qu'un seul en 2010, il reste nettement inférieur à celui présenté par les données officielles chinoises. La différence s'explique aisément par le nombre d'espaces qui ont ouvert sous l'appellation d'espaces de fabrication numérique dans le seul but de bénéficier des subventions étatiques alors qu'il s'agissait souvent d'espaces de bureaux, de cyber-cafés ou d'espaces de co-travail sans aucun équipement spécifique. Dans ce sens, la promotion des espaces de fabrication numérique, 众创空间, est un prolongement des politiques de créations de clusters urbains créatifs que mène le gouvernement central depuis plusieurs années avec des résultats mitigés. Souvent, elles ont résulté en opérations immobilières rentables, sans toutefois attirer les entreprises innovantes que ces nouveaux espaces devaient accueillir (Renaud et al., 2016).

Il semblerait toutefois qu'une part de la confusion vienne du terme chinois d'espace de fabrication numérique lui-même, utilisé pour désigner toutes sortes d'acteurs et de lieux ayant une activité liée aux nouvelles technologies. Le terme 创客 (chuangke) a été créé à l'occasion du Beijing Maker Carnival pour distinguer les makers des 黑客 (Heike), c'est-à-dire des hackeurs, qui sont associés au piratage informatique. En chinois le caractère 创 (chuang), signifie à la fois "commencer ", « initier » et "créer ». Il est utilisé pour composer des mots tels qu'innovation: 创新 (chuangxin), créativité : 创意 (chuangyi) ou fonder une entreprise, et startup : 创业 (chuangye), qui peut également se dire 初创 (chuchuang). L'utilisation de ce même caractère 创donne au terme faiseur 创 客 (chuangke) une flexibilité qui lui permet d'être employé dans une variété de circonstances se rapportant à la fabrication, la créativité, l'innovation ou l'entreprenariat. Ainsi, certains ingénieurs rencontrés à Shenzhen ont dit travailler 
dans un 创客空间 (espace de fabrication numérique) en parlant simplement de l'entreprise qui les employait.

On peut voir dans cette polysémie le reflet d'une interprétation chinoise du mouvement du faire, qui aurait pu être étouffée par l'ampleur des subventions et par le fait qu'à Shenzhen il y a finalement beaucoup plus d'incubateurs, d'accélérateurs de «jeunes pousses» d'entreprises, et d'espaces de co-travail que de vrais espaces de fabrication numérique. D'après David Li, le fondateur de Xinchejian (新车间), «la grande différence avec les autres pays comme les États-Unis ou la France, c'est que la Chine est toujours un pays en développement. Les gens ne regardent pas le mouvement du faire d'un point de vue politique, mais comme une opportunité de business. La plupart des espaces de fabrication ont été créés dans l'idée d'en faire des entreprises ou des incubateurs. Peu existent uniquement pour le loisir, comme le nôtre» (Tual, 2016). La Chine a fondé ce développement sur l'export de produits manufacturés. À Shenzhen et ses environs, où le mouvement du faire a trouvé un écho considérable, une grande partie des entreprises s'est spécialisée dans la sous-traitance de produits électroniques. Ainsi, l'éthique du faire, selon laquelle chacun devrait être capable de maîtriser la technologie grâce au partage des connaissances ainsi qu'à une pratique personnelle, est plus facilement accueillie car la production fait déjà partie du quotidien et que n'importe quel composant est immédiatement accessible. Il semblerait donc que la politique du gouvernement ait surtout contribué à attirer l'attention mondiale sur une ville dont l'histoire et l'organisation du territoire faisaient déjà un endroit privilégié pour le développement d'une culture du faire "aux caractéristiques chinoises" (Lindtner, 2015).

\section{Shenzhen et le Delta : réformes gouvernementales et dynamiques locales}

Shenzhen est une ville côtière du delta de la Rivière des Perles au Sud-Est de la Chine, située dans la province du Guangdong à la frontière de la région administrative spéciale de Hong Kong. À la fois assez éloignée du centre politique de Pékin et proche de la colonie anglaise ouverte sur le système économique mondial, la province a été la première à bénéficier des réformes expérimentales d'ouverture des années 1980. Grâce à la création des trois zones économiques spéciales de Shenzhen, Zhuhai et Shantou, puis à l'extension progressive de ces politiques de décentralisation économique et fiscale à l'ensemble du Delta, le bassin manufacturier du Guangdong s'est développé à partir d'une industrie d'assemblage tournée vers l'export, jusqu'à acquérir, dans les technologies numériques, une des plus grandes capacités productives mondiale.

Dans la province, les politiques volontaristes du pouvoir central sont en réalité entrées en synergie avec des dynamiques historiques et un régime de propriété foncière hérité, que certains auteurs nomment le "modèle d'urbanisation du Guangdong " (Chung et Unger, 2013). Cette rencontre a entraîné une concentration des industries à haute valeur ajoutée à Shenzhen, tandis que les industries d'assemblage étaient progressivement délocalisées dans le reste du Delta (Sanjuan, 1996). Renonçant aux expropriations massives nécessaires aux grands travaux d'aménagement, le gouvernement autorisa les comités villageois à conserver une partie de leurs terres afin de s'assurer de leur coopération. "Officiellement encouragés à développer une industrie et des activités secondaires en autosuffisance (zili gengsheng) » au temps des 
«équipes de production» maoïstes des années 1970, c'est dans la continuité de cette industrialisation rurale que tous se mirent à construire des bâtiments industriels destinés à la location, « chaque village rivalisant avec les autres dans l'idée de devenir auto-suffisant " (Chung et Unger, 2013). Tandis que les prix fonciers augmentaient dans le centre de Shenzhen, les villages extérieurs devenaient donc plus attractifs pour les entreprises hong-kongaises. S'y est alors développé un dense tissu de petites usines d'assemblage à faible valeur ajoutée destiné à l'export dans des secteurs tel que l'électroménager, l'ameublement, la petite électronique ou encore la transformation plastique.

\section{Illustration 2 - Vue satellite de l'urbanisation du Delta depuis 1988}

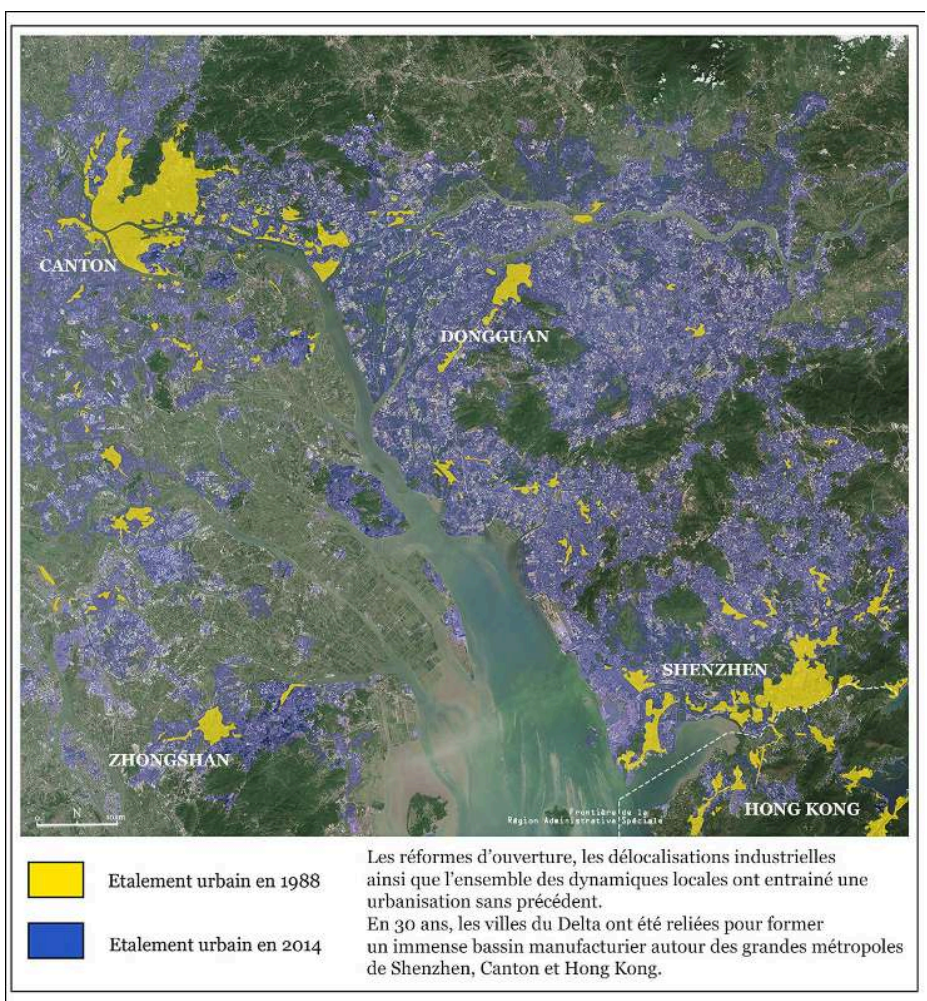

Source : Images satellites du NASA Earth Observatory par Joshua Stevens, à partir de données Landsat du US Geological Survey. Auteur : J. Nawrocki, 2020.

En marge de cette économie de sous-traitance, de nombreuses entreprises, souvent structurées autour de réseaux familiaux, se sont alors lancées dans la fabrication de produits électroniques destinés à la population locale. Conçus pour être vendus au prix le plus faible, ceux-ci étaient d'abord copiés sur les produits occidentaux avant que des adaptations ne soient apportées pour correspondre à des besoins spécifiques et cibler les marchés de niche. Issues de l'industrie de la contrefaçon, où les objets à copier étaient d'abord démontés, étudiés, avant d'être reproduits, plus vite et moins cher, ces itérations successives ont parfois donné lieu à de véritables innovations. En Chine, ce secteur a été désigné par le jeu de mot Shanzhai (山寨), qui signifie «fortin montagnard" ou "repaire de brigands en montagne», et évoque l'idée d'une innovation locale qui volerait aux riches pour donner aux pauvres. Certaines des entreprises les plus compétitives sont ensuite devenues de grandes firmes 
internationales, comme Tecno, aujourd'hui leader sur le marché africain de la téléphonie mobile.

Autour de Shenzhen, ce mode de production a pu se développer dans ce secteur quand la société taïwanaise Médiatek (MTK) a mis en vente en 2005 un processeur ouvert, sans droits de propriété intellectuelle. Étant le composant le plus complexe, celui-ci a permis à n'importe quel producteur de développer facilement son propre téléphone, sans coûts de recherche \& développement prohibitifs à l'entrée sur le marché. La filière s'est alors structurée autour de véritables clusters au sein desquels chaque entreprise était spécialisée dans la fabrication de composants particuliers. Dans leur article intitulé "The business model of a shanzhai mobile phone firm in China ", Jin-li Hu et Hang Zhu (Hu et al., 2011) décrivent également comment les entreprises de différents domaines d'activité, tels que le design industriel, l'assemblage final ou la distribution, coopèrent pour former une chaîne d'approvisionnement rapide et efficace. D'après les auteurs, un cluster de fabricants Shanzhai est ainsi capable de lancer un nouveau produit tous les 2mois, " alors qu'une marque internationale nécessitera 6 à 12 mois de recherche et développement » avant de pouvoir démarrer la production.

La proximité spatiale, à la fois entre producteurs et avec la clientèle, ainsi que la concurrence sur les délais de mise sur le marché ont par ailleurs favorisé un mode de production synchronisant les phases de conception et d'assemblage au sein d'un processus itératif. La pression permanente pour lancer les produits le plus vite possible et à moindre coût, à cause de la concurrence avec les grandes marques internationales et au sein même de l'écosystème productif Shanzhai a entrainé la suppression des phases de test des produits avant leur lancement sur le marché. Afin de s'adapter à la demande, ils ont donc été pensés dès le départ pour pouvoir être assemblés de la façon la moins chère, la plus efficace et la plus rapide possible. Le design a été subordonné à la fabrication, et les acteurs avaient donc connaissance des différentes possibilités dont ils disposaient. Contrairement à un système de sous-traitance classique, dans lequel les phases de conceptions et de fabrication sont cloisonnées, la proximité spatiale permettait alors de revenir facilement sur le design initial si celui-ci s'avérait inadapté, ou si une meilleure solution avait simplement été pensée en cours de production.

Des recherches récentes ont caractérisé le Shanzhai comme un "nouveau modèle d'innovation ouverte", basé sur un "travail de composition à partir d'éléments existants » (Renaud, 2017) et un design dérivé des procédés de fabrication. "Au contact des chaines de fabrication, le travail de conception donne lieu à des assemblages inédits qui dépendent directement des ressources et technologies disponibles » (Renaud, 2017), sous l'influence déterminante du marché local. Ce mode de production par assemblage a donc été une adaptation pragmatique à la concurrence, ainsi qu'une manière d'utiliser le plus efficacement possible un tissu industriel constitué au cours des trois dernières décennies. Désormais, ces usines issues de la contrefaçon forment à Shenzhen un écosystème concurrentiel original, dans lequel la collaboration, l'échange de connaissances, de ressources et de procédés rappelle les modèles d'innovation ouverte qui ont été adoptés par les programmeurs informatiques américains dans les années 1990, «à ceci près que les biens échangés ne sont pas immatériels (code, connaissances ou savoir-faire), mais des objets réels » (Renaud, 2017) qui peuvent être facilement intégrés à d'autres projets. 


\section{Une région planifiée autour de l'innovation numérique}

\section{La scène du faire}

Plus qu'un type d'organisation industrielle, le Shanzhai a été décrit par les médias comme un phénomène culturel, une innovation pirate pouvant être appréhendée par son état d'esprit subversif ou par son style. Cette culture, en défendant également une créativité basée sur le contournement des droits de propriété intellectuelle, est devenue le ciment de ce qui pourrait être appelé une "scène» du faire, au sein de laquelle elle fait écho à la philosophie du logiciel libre occidentale. L'idée de scène engloberait alors non seulement la communauté des makers définie par une culture et une identité posée a priori, mais aussi tout un ensemble d'activités et d'acteurs aux intérêts parfois contradictoires, ainsi que leur ancrage dans des «espaces culturels préexistants, attendant d'être occupés et définis » (Straw, 2006).

Reprendre cette notion à Shenzhen, d'abord élaborée par Will Straw comme un modèle analytique pour étudier de façon unifiée la diversité des pratiques liées aux courants musicaux à Montréal, aide ainsi à dépasser les difficultés méthodologiques résultant de la diversité d'espaces et de pratiques qui ne pouvaient pas toujours être rattachées au mouvement du faire mais façonnaient tout de même une culture urbaine cohérente. L'idée de scène permet d'explorer « la dynamique qui anime l'ensemble » (Arrivé, 2019) et de comprendre l'attractivité de la ville qui ne peut s'expliquer par les seules politiques d'aménagement de "clusters créatifs industriels " et "d'innovation de masse ", ni par les subventions massives accordées depuis 2015. Celles-ci auraient même tendance à étouffer le mouvement, en exacerbant la concurrence ainsi que la pression foncière, obligeant finalement les espaces de fabrication numérique à adopter des stratégies de développement commercial. Elles vont donc à l'encontre de certains principes fondateurs de l'idéologie du faire, comme l'autonomie et le bricolage de loisir dans des espaces ouverts aux amateurs, sans but lucratif. Ces politiques de soutien à l'innovation, qui menacent l'existence des espaces de fabrication et sapent ainsi ellesmêmes les bases du modèle qu'elles entendent promouvoir ont pourtant alimenté l'effervescence d'une « scène ». Elles ont apporté non seulement des moyens financiers, mais ont surtout attiré l'attention internationale sur la ville, insisté sur son potentiel, tout en se greffant sur des dynamiques locales qui ont continué à évoluer par des voies détournées. Pour ne prendre que quelques exemples, on peut citer la fermeture de l'espace Labzero qui ne parvenait plus à payer son loyer, mais dont la communauté de makers s'est maintenue en ligne, pour devenir le cœur d'une scène plus vaste, vivant au rythme d'événements organisés par les groupes de conversation du réseau WeChat. On peut également mentionner le site de vente en ligne Taobao et son infrastructure de livraison subventionnée par le gouvernement, qui permet un accès encore plus rapide aux divers composants nécessaires au prototypage. Le déclin du marché de composants électroniques de Huaqiangbei, symbole du Shanzhai et de la culture du faire à la chinoise, est donc compensé par une accélération des flux qui rapprochent encore plus la fabrication de la conception. Enfin, la promotion officielle d'un mouvement "d'espaces de fabrication numérique de masse", slogan pourtant contradictoire, a entraîné la construction d'un discours décrivant le Shanzhai comme un mode de production en code source ouvert. Alors qu'il était perçu de façon négative par le gouvernement, comme une industrie de contrefaçon à éradiquer, il est désormais mis 
en avant avec fierté, en tant que culture à la base d'un nouveau modèle d'innovation de masse, alternatif à la délocalisation.

\section{Les espaces de fabrication numérique, des espaces hybrides au cœur de la scène}

Dans le cas de l'émergence de la scène du faire de Shenzhen, encore plus que dans celui des scènes musicales (Straw, 2006) ou même informatiques comme celle du bitcoin (Arrivé, 2019), le contexte territorial ainsi que sa densité historique semblent déterminants. L'écosystème productif de l'ensemble de la région du Guangdong donne de l'ampleur au mouvement, en conférant une dimension industrielle au bricolage des makers. Le développement économique de la ville et l'effervescence qui en découle alimentent l'enthousiasme qui fait vivre la scène autour des cultures shanzhai et hackeur. Celle-ci, en retour, contribue à l'attractivité de la ville, qui n'est pas le simple résultat de la planification et des politiques successives du gouvernement central.

La scène du faire de Shenzhen semble donc avoir ceci de particulier qu'elle est diffuse dans tout l'espace urbain, en se situant à l'intersection entre un système de production basé sur des grandes entreprises liées à l'État et le système plus indépendant des makers, dont beaucoup sont des étrangers, mais qui est également englobé dans la politique économique de Pékin. Cette intersection est matérialisée par le quartier de Huaqiangbei qui forme une centralité dans une ville articulée autour de différents districts relativement indépendants. Cet immense marché de composants électroniques et de produits shanzhai symbolise la spécialisation de la région dans la production numérique et évoque à la fois le bricolage à partir de composants bruts cher aux makers, et la réussite de grands groupes issus de la contrefaçon et plus largement de la politique d'ouverture des années 1980, comme Huawei ou Tecno. Bien que Huaqiangbei soit aujourd'hui concurrencé par les plateformes de vente en ligne, il reste la référence commune à tous les acteurs de la scène du faire. Au-delà du symbole culturel, il témoigne dans le centre même de l'ancienne zone économique spéciale d'une force productive héritée, offrant désormais une proximité inédite entre conception et assemblage de masse.

Dans cette configuration, les espaces de fabrication numérique dispersés dans l'ensemble de la ville peuvent être considérés comme les nœuds structurants de la scène, à l'articulation d'espaces physiques, virtuels, cognitifs, productifs, et relationnels en recomposition permanente. Ils sont des lieux de sociabilité, des centralités en ville, voire un premier point de chute pour les expatriés, tout en offrant un espace de travail, des outils et l'entraide d'une communauté. Par ce qu'ils incarnent en eux-mêmes, avec leurs salles dédiées aux machines et aux composants électroniques, ils sont aussi les catalyseurs d'une nouvelle culture urbaine numérique, des lieux hybrides, entre matériel et virtuel. Leur spécificité tient cependant au fait qu'à Shenzhen, ils servent avant tout de relais vers l'écosystème productif régional. 
Illustration 3 - Les espaces de fabrication numérique de Shenzhen et le développement économique vers l'Ouest de la ville

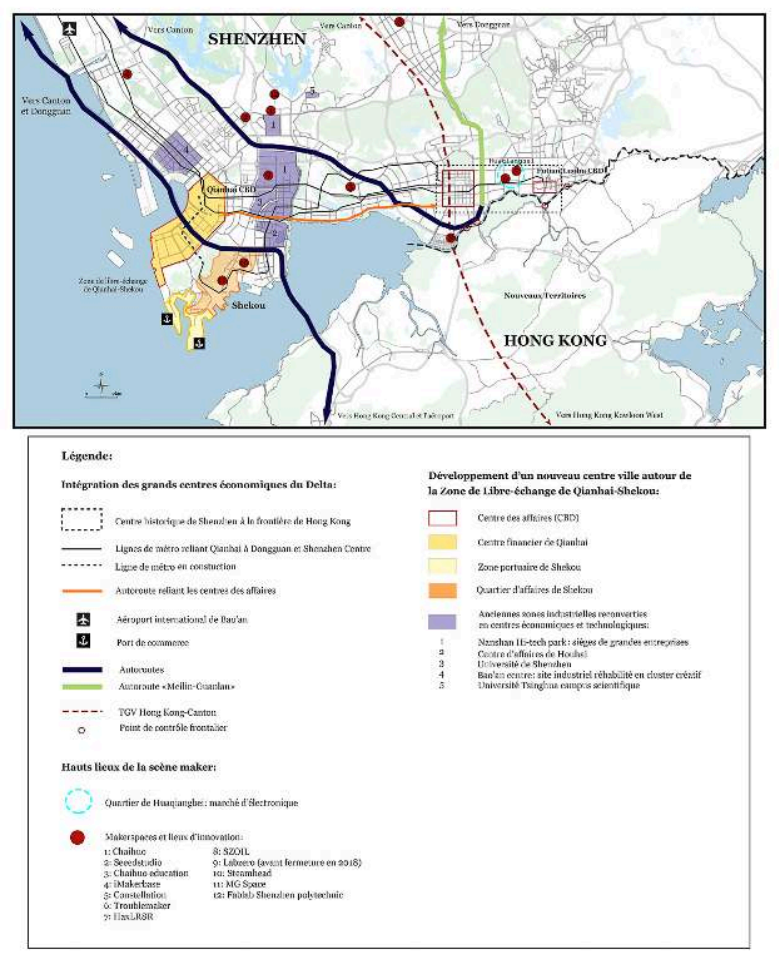

Sources : Relevés de terrain. Auteur : J. Nawrocki, 2020.

Derrière l'appellation « espace de fabrication numérique » (创客空间), utilisée de façon très large par le gouvernement chinois, cette étude traite en réalité de plusieurs types de «tiers-lieux » (Oldenburg, 1989) qui se distinguent essentiellement par leur modèle d'affaire, tels que les espaces de fabrication numérique, les laboratoires du faire, les incubateurs, les accélérateurs de «jeunes pousses» ou les espaces de co-travail. À Shenzhen, les deux premiers types de tiers-lieux restent plus communautaires et éducatifs tandis que les derniers ont uniquement une vocation de rentabilité; mais aucun de ces espaces n'est autogéré comme peuvent l'être certains des espaces de fabrication occidentaux à l'origine du mouvement. Malgré la différence de leurs activités, tous ont ici un but lucratif en proposant de faciliter à leurs membres l'accès aux ressources locales, voir régionales. Il serait donc possible de les désigner par le terme " plateforme du faire » (" maker hub») utilisé par l'entreprise SeeedStudio. Grâce à leurs contacts, ces espaces servent de liens entre les makers, les entrepreneurs ou les ingénieurs qui viennent à Shenzhen dans le but de fabriquer un prototype ou lancer une production, et les industries manufacturières locales. Comme l'ont montré les entretiens: "les industries de Shenzhen ont l'habitude de produire en masse pour l'exportation, et refusent de fabriquer de petites séries. Il faut alors du temps pour visiter toutes les usines de Dongguan et trouver celle qui est prête à prendre une petite commande. " Un des services proposés à Shenzhen par ces plateformes est donc de mettre en contact concepteurs et producteurs. Ce service bénéficie également aux propriétaires d'usines, selon Anson de l'espace X.Factory, puisqu'il «les aide à se reconvertir vers la production agile (Agile manufacturing). Leurs usines peuvent donc 
rester compétitives et s'adapter aux changements économiques sans avoir à investir pour automatiser toute la production et faire des économies de personnel ».

La production agile consiste à mettre en place, sur de petites séries, des cycles de développement itératif, en collaboration avec le client. Les phases d'assemblage doivent pouvoir s'adapter aux changements en cours de conception plutôt que de suivre un plan fixe comme dans la production de masse. À Shenzhen, ce modèle bénéficie de la disponibilité quasi-instantanée de n'importe quel composant, mais surtout de l'expérience acquise par les producteurs dans les procédés de fabrication industrielle. D'une part, beaucoup d'usines sont déjà organisées en réseaux collaboratifs dans l'industrie shanzhai et travaillent avec des procédés similaires. D'autre part, le savoirfaire acquis à travers les activités de sous-traitance permet désormais à leurs ingénieurs de proposer des solutions de fabrication et de participer au processus de conception. Grâce à une organisation qui accélère les échanges entre fournisseurs, producteurs et concepteurs, l'écosystème manufacturier de Shenzhen rendrait alors la production industrielle accessible à n'importe quel entrepreneur dépourvu de capacité d'investissements en activités de recherche et développement. Les dynamiques de la scène du faire favoriseraient quant à elles l'apprentissage des procédés de fabrication au sein des espaces de fabrication numérique, à travers les réseaux sociaux virtuels ou au cœur même des usines de la région. L'ensemble aboutirait finalement à une concentration spatiale de savoir-faire manufacturier ouvert et, d'après les discours officiels ainsi que certains auteurs (Lindtner et al., 2015 ; Fernandez et al., 2016 ; Renaud, 2017) à un nouveau modèle d'innovation par le faire, une industrie sous matériel libre de masse.

\section{Trois systèmes productifs pour un modèle d'innovation en matériel ouvert}

30 Afin de préciser l'organisation du bassin manufacturier de Shenzhen et de comprendre comment il pouvait permettre le développement d'une économie créative ainsi que la sortie d'un modèle d'assemblage à faible valeur ajoutée, selon les objectifs du $13^{\text {ème }}$ plan quinquennal, plusieurs projets industriels ont été analysés dans le cadre d'entretiens semi-directifs. Ces entretiens visaient à illustrer par des exemples concrets les différentes façons dont peut être exploité l'écosystème productif de Shenzhen. Les résultats de ce travail ont précisé en quoi le mouvement du faire et sa pratique du code source ouvert ne constituaient pas une rupture, mais plutôt une évolution du modèle productif hérité. Les projets analysés ont par ailleurs permis de souligner que ce qui était présenté comme un nouveau modèle d'innovation unifié était en réalité composé de plusieurs types d'organisations productives. Cette enquête de terrain en a identifié trois. En ce sens, le terme "d'écosystème », employé aussi bien par le gouvernement que par les chercheurs (Lindtner, 2015 ; Lindtner et al., 2015; Renaud, 2017) et les habitants de Shenzhen, semble effectivement approprié.

\section{La sous-traitance}

31 Tout d'abord, plusieurs entretiens ont montré que la sous-traitance d'assemblage classique constituait toujours le principal moteur de l'industrie deltaïque. Ce résultat s'accorde avec la thèse de Mei Wu sur Dongguan ( $\mathrm{Wu}, 2016)$, la ville voisine de Shenzhen où les acteurs interrogés font fabriquer l'essentiel de leur production. Son 
étude souligne les difficultés auxquelles font face les usines, telles que la pénurie de main-d'œuvre, la faible valeur ajoutée et la dépendance aux technologies étrangères. La plupart des entreprises sont en effet des fabricants d'équipements d'origine (FEO), liées par contrat de sous-traitance à des constructeurs pour lesquels elles produisent des pièces détachées. Celles-ci sont fabriquées d'après les critères de l'acheteur qui s'occupe de la conception grâce à d'importants investissements en activités de recherche et développement mais qui ne dispose pas des capacités d'assemblage en interne. De nombreux makers interrogés sont donc des consultants employés par des multinationales pour vérifier et superviser la production des FEO. L'enquête de terrain a toutefois montré qu'en parallèle de leurs activités, ces makers développaient des projets personnels en profitant des opportunités de la ville. Dans ce cas de figure, les espaces de fabrication offraient un lieu de travail personnel, les outils nécessaires ainsi qu'un accès quasi immédiat à tous les composants électroniques produits dans la région. Ils y concevaient ensuite leurs prototypes eux-mêmes, selon les méthodes du design itératif qui consistent à modifier et à améliorer un projet au fil des différentes itérations qui seront réalisées jusqu'au produit final.

\section{Le design itératif}

32 Pour être rentable, ce processus doit être le plus rapide et le moins cher possible. À Shenzhen, c'est la capacité à mettre en œuvre cette méthode de conception de manière optimale qui a attiré tous les acteurs rencontrés. Le design itératif représente donc un deuxième type d'organisation productive qui se développe en parallèle dans la région. Les entrepreneurs travaillent alors en étroite collaboration avec des FEO qui restent sous-traitants, puisqu'ils exécutent bien une commande, mais qui participent pleinement à l'amélioration du design final. Ils mettent à disposition leur savoir-faire et leurs réseaux, souvent constitués dans le cadre de l'industrie Shanzhai, tandis que l'achat des composants en ligne et la rapidité des services de transport accélèrent encore les délais d'exécution. Dans ce mode de production, les espaces de fabrication servent surtout de relais vers le reste de l'écosystème manufacturier et de lien avec une communauté d'entrepreneurs, sans que leur espace ne soit utilisé pour la production. Même pour concevoir le prototype, la fabrication est directement confiée aux usines d'assemblage. C'est par exemple de cette façon que procédait la "jeune pousse " Infinite Food, dont un des fondateurs nous a dit n'avoir aucun ingénieur dans son équipe, ses associés ne disposant que des connaissances basiques en design industriel. D’origine australienne, il était donc venu à Shenzhen afin de profiter du savoir-faire des usines de la région.

\section{Un « facilitateur de fabrication matérielle »}

Enfin, des entretiens ont permis de définir ce qui pourrait être une troisième forme d'organisation productive, qui s'inspire ouvertement de la culture Shanzhai et des pratiques du code source ouvert. Celle-ci peut être définie comme une plateforme, le «facilitateur de fabrication matérielle» (hardware enabler), qui travaille également selon la méthode du design itératif mais intègre directement l'ensemble des étapes pour faciliter («enable») la production. La plateforme dispose donc d'une équipe d'ingénieurs qui conçoit en interne des composants au code source ouvert. Ceux-ci peuvent ensuite être intégrés à toutes sortes de projets que "le facilitateur » aide à prototyper de façon adaptée à une future production de masse. Pour ce faire, la 
plateforme dispose d'une capacité de fabrication en petite série sur place, et d'un réseau d'usines avec lesquelles elle met ses clients en contact au moment d'augmenter la production, tout en prenant en charge les stocks et les premières livraisons. La plateforme dispose également d'un grand espace de fabrication permettant d'accueillir une communauté de membres et de créer ainsi un espace d'entraide et de partage. Elle propose par exemple de mettre en contact ses adhérents avec des makers qui ne travaillent sur aucun projet en particulier, ce qu'elle appelle les skills with demand. Ce service permet à des entreprises qui ne disposent pas d'équipes d'ingénieurs et ne souhaitent pas investir dans des activités de recherche et développement de travailler sur un nouveau produit. C'était par exemple le cas du manager d'une société de télécommunication finlandaise interviewé chez Seeedstudio qui souhaitait développer un projet d'internet des objets. Fournissant déjà à ses clients un réseau $5 \mathrm{G}$ avec un débit suffisamment puissant, l'entreprise cherchait à élargir sa gamme de services en offrant directement des moyens d'utiliser ce réseau, sans pour autant investir dans un nouveau pôle de recherche et développement.

L'idée du «facilitateur » est donc d'abaisser les barrières à l'entrée sur le marché de l'électronique en fournissant du matériel et des compétences libres de droit, ainsi qu'un accès privilégié au tissu manufacturier local. Comme dans la téléphonie Shanzhai, qui a innové à partir d'un processeur libre mis en vente par MTK, le «facilitateur » souhaite permettre à ses clients d'innover en se concentrant sur d'autres étapes du prototypage que celles consistant à concevoir et fabriquer les composants électroniques de base.

\section{Conclusion}

Dans le domaine de l'industrie électronique, le fait de fournir les plans de fabrication ainsi que le code source des logiciels pilotes ne suffit pas à rendre leur reproduction aussi accessible que dans la programmation où l'ouverture du code s'était vite avérée rentable. Il faut d'abord disposer des outils et des machines nécessaires, ce qui peut devenir un obstacle en raison de la complexité du contenu technologique et des quantités à produire. Considérer l'écosystème productif de Shenzhen comme un nouveau modèle d'innovation ouvert invite donc à appréhender l'idée d'une production sous matériel libre de masse comme un travail de "composition à partir d'éléments existants » (Renaud, 2017), reposant sur la synchronisation des phases de conception et d'assemblage. Il ne s'agirait pas tant d'ouvrir l'accès au code source que de faciliter celui à la fabrication, grâce à la proximité de tout un territoire manufacturier constitué au cours des dernières décennies et qui continue d'évoluer en lien avec les technologies numériques. Les FEO de la région ne produisent plus simplement des composants dont le design leur serait fourni par un client avec lequel ils signent un contrat d'exclusivité. Grâce à l'expérience acquise dans la fabrication au cours des dernières décennies, ils ne sont plus totalement dépendants des équipes de recherche et développement externes. Les sous-traitants interviennent donc avant la phase de conception qu'ils vont orienter en fonction des ressources technologiques mises à dispositions des concepteurs, puis au cours d'un travail de collaboration tout au long de l'assemblage du prototype. En inversant ainsi les étapes de production, ils remettent la fabrication au cœur du processus créatif et rejoignent un des principes fondateurs de la culture du faire et des makers. 
Après avoir su tirer parti de l'apparition de ce mouvement, ainsi que de l'industrie shanzhai initialement accusée de contrefaçon, le gouvernement de Pékin souhaite faire de Shenzhen et de son système productif un moteur du développement deltaïque. La ville se trouve donc au cœur d'un « corridor d'innovation " prévu pour relier Canton à Hong Kong à l'horizon 2025, dans le cadre du projet d'intégration de la " Grande Baie ». La région, qui s'est construite à partir d'une industrie d'assemblage à faible valeur ajoutée, évoluerait ainsi vers une économie de la connaissance, mais à partir d'un modèle d'innovation alternatif, basé sur la fabrication. En parallèle, la municipalité de Shenzhen continue toutefois de se tourner vers les services et la finance, en concurrence avec Hong Kong, notamment avec le nouveau centre des affaires de Qianhai à l'Ouest de la ville (Nawrocki, 2020). Il est donc permis de s'interroger sur l'évolution de ce modèle d'innovation et sur la cohérence des projets d'aménagement. L'écosystème d'innovation de Shenzhen est-il une simple étape de transition vers une économie immatérielle, basée exclusivement sur la production de données et d'informations, ou représente-t-il un modèle original susceptible d'être répliqué à l'échelle du Delta pour maintenir la croissance, comme le laisse sous-entendre le slogan Created in China 2025 ?

\section{BIBLIOGRAPHIE}

Arrivé E., 2019. Dynamique du fétichisme numérique : le cas bitcoin. Thèse de doctorat, Université Lumière Lion 2, sciences de l'information et de la communication, $382 \mathrm{p}$.

Arvanitis R., Zhao W, Qiu H and Xu J, 2006, Technological learning in six firms in southern China: success and limits of an industrialization model., Int. J. Technology management, $\mathrm{n}^{\circ}$ 36, p. 108-125.

Arvanitis R., Jastrabsky E., 2005. Un système d'innovation régional en gestation : Le cas du Guangdong. Perspectives chinoises, $\mathrm{n}^{\circ}$ 92, p. 14-28.

Beckouche P., 2017. La révolution numérique est-elle un tournant anthropologique ? Le Débat, $n^{\circ}$ 193, p. 153-66.

Beckouche P., 2019. Les nouveaux territoires du numérique : l'univers digital du sur-mesure de masse, Auxerre, Editions sciences humaines, $168 \mathrm{p}$.

Besson R., 2014. Capitalisme cognitif et modèles urbains en mutation. L'hypothèse des Systèmes Urbains Cognitifs. Territoire en mouvement Revue de géographie et aménagement. Territory in movement Journal of geography and planning, $\mathrm{n}^{\circ} 23-24$, p. 102-114.

Broca S., 2018. Matière et territoire dans la culture du logiciel libre. Géographie, économie, société, vol. $20, \mathrm{n}^{\circ} 1$, p. $15-32$.

Broca S., 2013. Utopie du logiciel libre. Paris, Éditions le passager clandestin, 288 p.

Cao C., 2004. L'industrie chinoise face au défi technologique : Les investisseurs étrangers sont les premiers pourvoyeurs de technologies. Perspectives chinoises, $\mathrm{n}^{\circ}$ 83, p. 4-17. 
Chung H., Unger J., 2013. The Guangdong model of urbanization: collective village land and making of a new middle class. China perspectives, vol. 3, p. 33-41.

Ferchaud F., 2017. Les communs urbains à l'épreuve du terrain : le cas des lieux de fabrication numérique. Netcom [En ligne], nº 31, p. 53-76. DOI: https://doi.org/10.4000/netcom.2628

Ferchaud F., Dumont M., 2017. Les espaces de fabrication et d'expérimentation numérique sontils des tiers-lieux : Une analyse de cas des fablabs à Rennes et à Toulouse. Territoire en mouvement [En ligne], $\mathrm{n}^{\circ}$ 34. DOI: https://doi.org/10.4000/tem.4203

Fernandez V., Puel G., Renaud C., 2016. The Open Innovation Paradigm: From Outsourcing to Open-Sourcing in Shenzhen, China. International Review for Spatial Planning and Sustainable Development, vol. 4, n' 4, p. 27-41.

Florida R., 2002, The rise of the creative class and how it's transforming work, leisure, community and everyday life. New York, Basic books, $416 \mathrm{p}$.

Gaschet F., Lacour C., 2007. Les systèmes productifs urbains : des clusters aux « clusties » /, Urban production systems: from clusters to clusties. Revue d'Économie Régionale \& Urbaine novembre, $\mathrm{n}^{\circ} 4$, p. 707-728.

Guibert G., 2016. La scène comme outil d'analyse en sociologie de la culture. L'observatoire, $\mathrm{n}^{\circ} 47$, p. 17-20.

Graezer Bideau F., Renaud C., 2020. Le maker, construction d'une figure politique de l'innovation en Chine urbaine. EspacesTemps.net [En ligne]. URL: https://www.espacestemps.net/articles/lemaker-construction-dune-figure-politique-de-linnovation-en-chine-urbaine

Haudeville B., Le Bas C., 2016. L'innovation frugale, paradigme technologique naissant ou nouveau modèle d'innovation ? Innovations, $n^{\circ} 51$, p. 9-25.

Hu J., Wan H., Zhu H., 2011. The business model of a shanzhai mobile phone firm in China. Australian journal of business and management research, vol. $1, \mathrm{n}^{\circ} 3$.

Hussler C., Burger-Helmchen T., 2016. Inversée vous avez dit inversée ? Une typologie stratégique de l'innovation inversée. Revue française de gestion, $\mathrm{n}^{\circ}$ 255, p. 105-119.

Keane M., 2004. Brave New World: Understanding China's Creative Vision. International Journal of Cultural Policy, vol. 10, n 3, p. 265-279.

Keane M., 2006. Created in china: the new catch up strategy. Proceedings international communication association, development and intercultural communication panel [En ligne]. URL: http:// eprints.qut.edu.au

Keane M., 2010. Reclaiming China's former soft power. Journal of the oriental society of Australia, $n^{\circ} 42$, p. 50-65.

Lazzarato M., Negri A., 1991. Travail immatériel et subjectivité. Futur antérieur, nº 6.

Liefooghe C., 2010. Économie créative et développement des territoires : enjeux et perspectives de recherche, Abstract. Innovations, $n^{\circ} 31$, p. 181-197.

Liefooghe C., 2018. Les tiers-lieux à l'ère du numérique : diffusion spatiale d'une utopie socioéconomique. Géographie, économie, société, vol. 20, n 1, p. 33-61.

Lindtner S. Li D., 2012. Created in China : the makings of China's hackerspace community. Interactions [En ligne], n 12, 18 p. DOI: https://doi.org/10.1145/2377783.2377789 
Lindtner S., 2015. Hacking with Chinese characteristics: the promise of the maker movement's against China's manufacturing culture. Science, technology \& human value [Online], $\mathrm{n}^{\circ}$ 40, p. 1-26. DOI: https://doi.org/10.1177/0162243915590861

Lindtner S., 2014. Hackerspaces and the Internet of Things in China: How Makers Are Reinventing Industrial Production, Innovation, and the Self. China Information, vol. 28, n² 2, p. 145-67.

Lindtner S., Greenspan A., Li D., 2015. Designed in Shenzhen: Shanzhai Manufacturing and Maker Entrepreneurs. Aarhus Series on Human Centered Computing, vol. 1, $\mathrm{n}^{\circ} 1,12 \mathrm{p}$.

Moilanen J., 2012. Emerging hackerspaces - Peer-production generation. $8^{\text {th }}$ International Conference on Open Source Systems (OSS), Hammamet, Tunisia., p. 94-111

Monnier J-M., Varcellone C., 2014. Le capitalisme cognitif, nouvelle forme de capitalisme ?, Problèmes économiques, Hors-série, $\mathrm{n}^{\circ}$ 5, p. 117-120.

Nawrocki J., 2020. Carte à la une. Shenzhen au cœur d'un corridor d'innovation dans le Delta de la rivière des Perles. Géoconfluences [En ligne]. URL: http://geoconfluences.ens-lyon.fr/ informations-scientifiques/a-la-une/carte-a-la-une/shenzhen-delta-riviere-perles

O'Connor J., Xin G., 2006. A New Modernity? The Arrival of 'Creative Industries' in China. International Journal of Cultural Studies, vol. 9, $\mathrm{n}^{\circ}$ 3, p. 271-283.

Radjou N., Prabhu J., Ahuja S., Roberts K., 2012. Jugaad innovation : think frugal, be flexible, generate breakthrough growth. New Jersey, Wiley, 288 p.

Renaud C., 2017. L'assemblage comme forme d'innovation. Techniques \& Culture, n’ 67, p. 100-115.

Renaud C., Fernandez V., Puel G., 2016. Changing the culture of innovation in Shanghai: Open innovation spaces. GeoInnov.

Sanjuan T., 1996. Le delta de la Rivière des Perles : développement et construction régionale dans la province du Guangdong (République populaire de Chine - 1980-1992). Thèse de Géographie, Université Paris 4.

Sanjuan T., Trolliet P., 2010. La Chine et le monde chinois : une géopolitique des territoires. Paris, Armand Colin, $384 \mathrm{p}$.

Straw W., 2004. Cultural scenes Society and leisure. Vol. 27, n² 2, p. 411-422.

Straw W., 2006. Scenes and sensibilities. Revista da Associaçao National dos programas de posgraduaçao em comunicaçao, vol. $16, \mathrm{n}^{\circ} 2$.

Wu M., 2016. Industrialisation and regional development in Guangdong province, China: A comparative study of two models in the post-1978 period. $\mathrm{PhD}$ thesis SOAS University of London.

Yu H., 2017. Editorial - Au-delà du e-commerce. Perspectives chinoises [En ligne], vol. 4, p. 3-9. URL : http://journals.openedition.org/perspectiveschinoises/7884

Yu H., 2017. Pivot to internet plus: Molding China's Digital Economy for economic restructuring? International Journal of Communication, $\mathrm{n}^{\circ} 11$, p. 1486-1506.

\section{RÉSUMÉS}

Ce travail interroge la façon dont les cultures numériques produisent de l'espace géographique et comment celui-ci influence en retour l'innovation technologique. Il montre comment le mouvement du faire de Shenzhen s'est développé dans un bassin manufacturier hérité, en faisant écho à la culture shanzhai locale. Ce mouvement a permis de conceptualiser un nouveau modèle 
d'innovation ouverte inspiré des pratiques du logiciel libre, qui alimente l'effervescence d'une scène du faire, engendrant ainsi de nouvelles dynamiques productives et spatiales.

This work investigates how digital cultures produce geographic space and how this one influences back technological innovation. It shows how Shenzhen's maker movement has developed in an inherited manufacturing region, echoing local shanzhai culture. This movement permitted to conceptualize a new open innovation model inspired from open-source ideology, which fuels the effervescence of a maker's scene, thus producing new spatial and productive dynamics.

\section{INDEX}

Mots-clés : makerspace, Shenzhen, shanzhai, numérique, innovation

Keywords : makerspace, Shenzhen, shanzhai, digital, innovation

Thèmes : Sur le Champ - Sur le Terrain

\section{AUTEUR}

\section{JULIA NAWROCKI}

Julia Nawrocki, julia.nawrocki@protonmail.com, est doctorante à l'Université Paris 1 Panthéon Sorbonne. Elle a récemment publié :

Nawrocki J., 2020. Carte à la une. Shenzhen au cœur d'un corridor d'innovation dans le Delta de la rivière des Perles. Géoconfluences [En ligne]. URL: http://geoconfluences.ens-lyon.fr/ informations-scientifiques/a-la-une/carte-a-la-une/shenzhen-delta-riviere-perles 